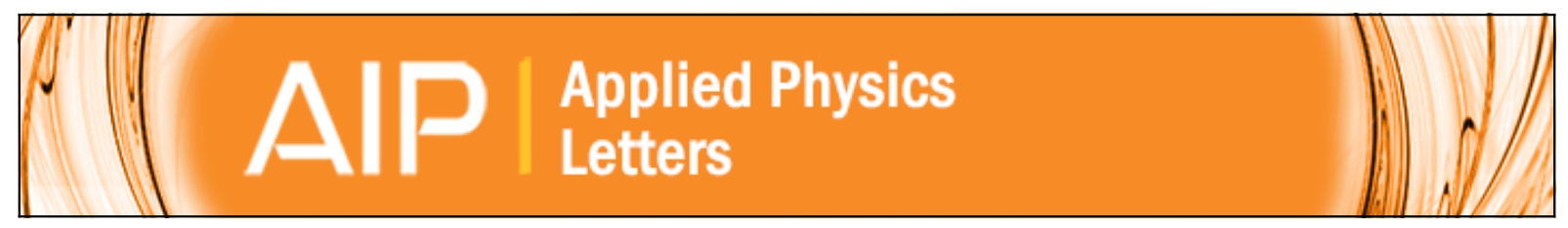

Measurement of optical mode loss in visible emitting lasers

P. C. Mogensen, P. M. Smowton, and P. Blood

Citation: Applied Physics Letters 71, 1975 (1997); doi: 10.1063/1.119759

View online: http://dx.doi.org/10.1063/1.119759

View Table of Contents: http://scitation.aip.org/content/aip/journal/apl/71/14?ver=pdfcov

Published by the AIP Publishing 


\title{
Measurement of optical mode loss in visible emitting lasers
}

\author{
P. C. Mogensen, a) P. M. Smowton, and P. Blood \\ Department of Physics and Astronomy, University of Wales Cardiff, Cardiff CF2 3YB, \\ Wales, United Kingdom
}

(Received 23 June 1997; accepted for publication 12 August 1997)

\begin{abstract}
We describe a technique for direct measurement of the passive optical mode loss of a semiconductor laser or similar semiconductor waveguide structure, based upon measurement of the attenuation of optically excited luminescence in the guided mode as a function of distance traveled along the passive guide. A spectrometer is used to select luminescence in the low energy tail of the spectrum which is subject to very little reabsorption. We have applied the method to a series of highly strained GaInP quantum well laser structures and observe an increase in the mode loss from $9.9 \mathrm{~cm}^{-1}$ for $1 \%$ strain to $46 \mathrm{~cm}^{-1}$ for $1.7 \%$ strain. This correlates with the appearance of clustered regions in the highly strained wells observed by transmission electron microscopy (TEM). (C) 1997 American Institute of Physics. [S0003-6951(97)03140-9]
\end{abstract}

The threshold current of a laser diode is determined primarily by the total optical loss from the lasing mode. Through the threshold condition and the gain-current relation the loss fixes the intrinsic recombination current and the positions of the electron and hole quasi-Fermi levels, which in turn control carrier leakage from the active region and other carrier loss processes. The total optical loss comprises the loss of light from the ends of the laser into the outside world and the loss of light from the guided mode as it propagates along the waveguide due to scattering and free carrier absorption. ${ }^{1}$ The end loss of a Fabry-Perot laser can be calculated using the Fresnel equation for the facet reflectivity and the modal loss is usually measured from the variation of external differential efficiency above threshold as a function of cavity length. ${ }^{2}$ Because of the need to use lasers of different length, this measurement is time consuming and subject to errors from random variations in properties of individual chips. It is therefore usual to assume typical values of mode loss for a particular type of laser and material system, based on a limited number of measurements. This approach is satisfactory when the structural integrity of the waveguide can be guaranteed and the dominant loss mechanism is the intrinsic effect of optical scattering by free carriers. However, there are situations where there may be significant contributions to the loss by scattering by structural defects and when measurement of the optical loss is an important aspect in the characterization of a device. In some cases a high modal loss may prevent the structure from operating as a laser. High modal loss may arise in structures with components under high strain, e.g., GaN-based structures for blue-emitting devices. While the differential efficiency method can be used to measure modal loss, this is subject to a number of practical and fundamental difficulties ${ }^{3}$ and does presuppose the existence of working lasers. It cannot be used to determine whether a high optical mode loss is the reason for the failure of a particular laser structure. In this paper, we describe a method of measuring the passive mode loss in a semiconductor waveguide without the need to have a working laser; in fact, no device processing is required. We have applied this

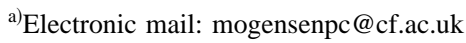

method to red-emitting AlGaInP structures with quantum wells grown at a high lattice mismatch with respect to the substrate. First, we describe the device structures and their laser performance then give an account of the measurement of modal loss and its application to these structures, not all of which function as lasers at room temperature.

The device structures were single quantum wells in identical separate confinement heterostructures. Three structures were grown with $\mathrm{Ga}_{x} \mathrm{In}_{1-x} \mathrm{P}$ wells of $100 \AA$ nominal width and with compositions $x=0.38,0.32$, and 0.28 corresponding to lattice mismatches of $1 \%, 1.4 \%$, and $1.7 \%$ respectively with respect to the lattice matched waveguide core and cladding of $\left(\mathrm{Al}_{0.4} \mathrm{Ga}_{0.6}\right)_{0.52} \mathrm{In}_{0.48} \mathrm{P}$ and $\left(\mathrm{Al}_{0.7} \mathrm{Ga}_{0.3}\right)_{0.52} \mathrm{In}_{0.48} \mathrm{P}$, respectively. The structures were grown by MOCVD on $n$-type [100] GaAs substrates misoriented $10^{\circ}$ off axis towards the [111]. The nominal compositions quoted above were confirmed by measurement of the optical absorption edges of the components of the structure by photovoltage spectroscopy.

When processed into $50 \mu \mathrm{m}$ oxide isolated stripe lasers and operated in a pulsed mode at a frequency of $1 \mathrm{kHz}$ and a pulse width of $300 \mathrm{~ns}$, the $1 \%$ and $1.4 \%$ strain devices lased at room temperature while the $1.7 \%$ strain device only lased up to $250 \mathrm{~K}$. At $200 \mathrm{~K}$, the threshold currents increased with increasing strain, with threshold currents for $750 \mu \mathrm{m}$ long lasers of 350 and $875 \mathrm{~mA}$, for the $1.0 \%$ and $1.7 \%$ strain samples, respectively. Transmission electron microscopy (TEM) examination showed that at $1.7 \%$ strain the quantum well and adjacent waveguide core had significant structural imperfections, and we wished to determine their effect on the modal optical loss and hence the threshold behavior. Full details of the structural investigations will be published in due course. Here we describe the technique developed to measure optical mode loss. It is of general applicability and could be particularly relevant to the study of nitride-based laser structures.

The method is based upon measurement of the attenuation of optically-excited luminescence in the guided mode of the structure as a function of distance traveled along the passive guide. A spectrometer is used to select luminescence in the low energy tail of the spectrum, which is subject to very little reabsorption by the well. This matter is examined in more detail below. A schematic diagram of the experi- 
(a)

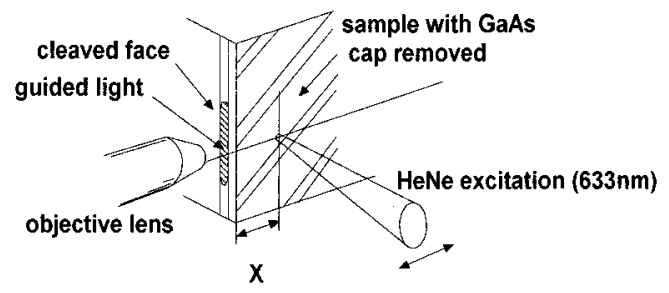

to spectrometer

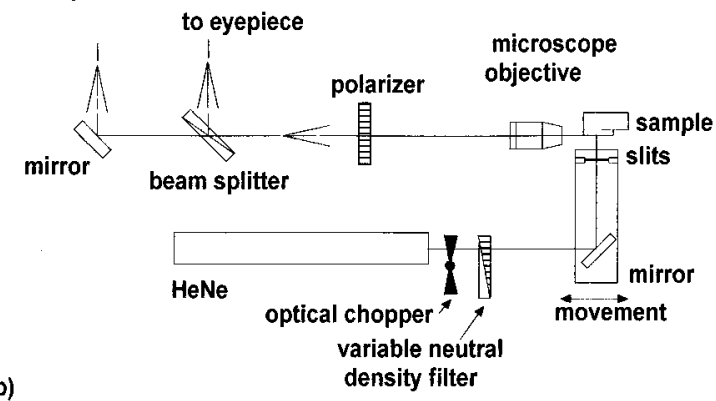

FIG. 1. A schematic diagram of (a) the sample geometry and (b) the experimental layout for the modal loss measurement.

mental arrangement is shown in Fig. 1, where Fig. 1(a) shows the relative position of the sample collection optics and pump beam. Similar sample and pump laser geometries are used for optical gain measurements. ${ }^{4}$ Figure 1(b), indicates the layout of the experimental apparatus. A chopped beam of light from an $\mathrm{HeNe}$ laser at $633 \mathrm{~nm}$ is directed onto the surface of a laser wafer with the GaAs top cap removed by wet etching. The beam passes through a pair of slits close to the sample to reduce the beam width to less than $0.5 \mathrm{~mm}$. This $633 \mathrm{~nm}$ light is only absorbed in the well region where it excites luminescence, and a typical spectrum is shown in Fig. 2. The part of this luminescence which is guided along the waveguide to emerge from a cleaved face of the wafer is collected by an objective lens and spatially filtered using the horizontal and vertical input slits of the spectrometer which were set to pass radiation at a wavelength in the low energy tail of the spectrum, as indicated in Fig. 2. The light is detected by a cooled GaAs photomultiplier tube connected to a

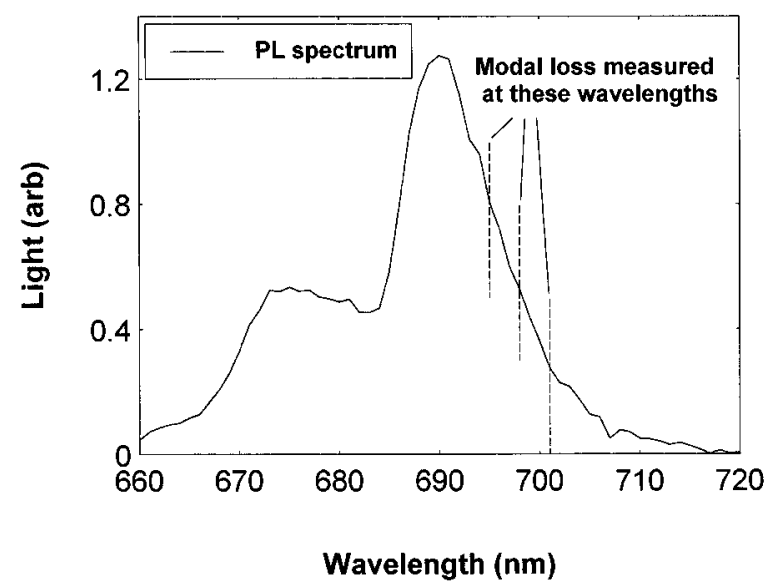

FIG. 2. Photoluminescence spectrum for a compressive strained GaInP quantum well laser structure, with dashed lines showing the different measurement wavelengths for the mode loss.

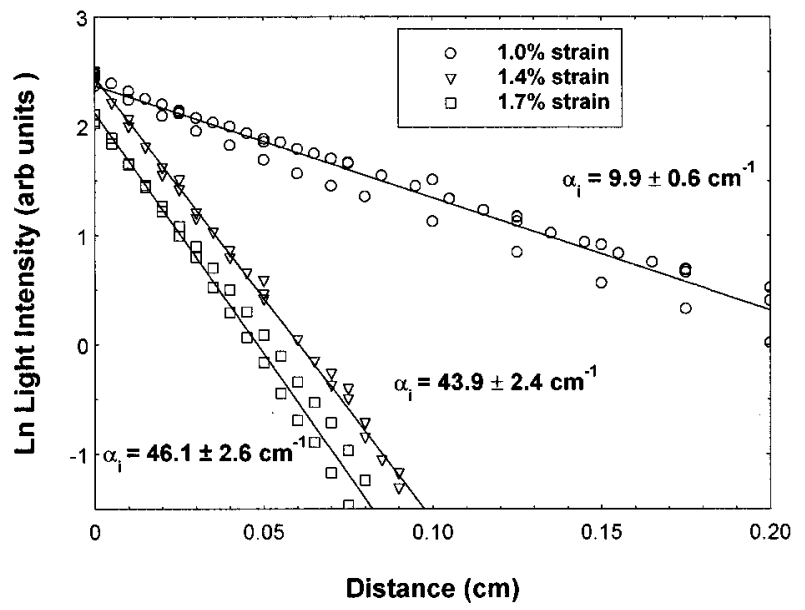

FIG. 3. Plot of the logarithm of PL intensity in the guided mode emerging from the edge of the wafer as a function of the distance " $X$ " traversed by the excitation beam. The calculated mode loss obtained from the gradient and the uncertainty in this quantity is given for each sample.

lock-in amplifier. The eyepiece shown in Fig. 1(b) is used to align the sample and the intensity of the pump source can be varied with the neutral density filter. The intensity of this guided radiation is measured as a function of distance of the excitation spot from the cleaved edge of the wafer, denoted by " $X$ " in Fig. 1(a). Plots of the logarithm of the photomultiplier signal versus distance are shown in Fig. 3 and are linear for all three structures yielding the values given for the corresponding absorption coefficients along with an uncertainty of two standard errors of the mean.

The light, which is guided along the waveguide, could be subject to reabsorption by the quantum well in addition to the losses due to free carrier absorption and scattering usually experienced by the guided mode in a pumped laser at and above threshold, when the well is providing gain rather than absorption. This reabsorption is negligible in this experiment for the following reason. The radiation is detected in the tail of the photoluminescence emission spectrum corresponding to the low energy edge of the absorption spectrum where the absorption coefficient is small. Furthermore, the optical confinement factor acts to scale down any residual absorption in the well by a factor of approximately 50 . To confirm that self-absorption is negligible, we have measured the modal loss at three different wavelengths shown by the dashed lines on Fig. 2. If self-absorption was making a contribution, we would expect this to be stronger as the detection wavelength is decreased to move up the absorption edge of the well. However, adjusting the detection wavelength from 695 to $701 \mathrm{~nm}$ does not produce any significant changes to the resulting decay constants. The equipment has been reassembled and the experiment repeated producing the same results within two standard errors.

The experimental results derived from Fig. 3 show that the modal absorption increases with increasing lattice mismatch. This correlates with TEM observations which show that the quantum well material tends to form into clusters at high compressive strain. We conclude that this clustering is responsible for the increased mode loss, up to about $44 \mathrm{~cm}^{-1}$ in the highest strain structure. In a working laser at threshold there will be an additional contribution to the mode loss of 
about $5 \mathrm{~cm}^{-1}$ due to the free carriers injected at threshold. Nevertheless, the experiments described here show that there is a significant increase in modal loss of about $35 \mathrm{~cm}^{-1}$ in a laser structure grown at $1.7 \%$ compressive strain compared with a $1 \%$ strain structure. This increase in optical loss must be an important factor in the failure of the $1.7 \%$ strain structure to work as a laser at room temperature.

We have described a technique for the measurement of optical mode loss in semiconductor laser structures, which does not require a working laser and can be applied directly to an as-grown wafer with a cleaved edge. The results obtained for highly strained GaInP quantum well structures show a correlation between an increase in mode loss and the appearance of clustered regions in the well observed by TEM. The technique should be of value for study of other laser structures subject to structural imperfections such as those grown in the GaN system.

The authors thank the Engineering and Physical Sciences Research Council for a studentship in association with EPI, Ltd. and Paul Hulyer for fabrication of the lasers. Transmission electron microscopy measurements were performed at the Manchester Materials Science Centre by S.A. Hall and U. Bangert.

${ }^{1}$ L. A. Coldren and S. W. Corzine, Diode Lasers and Photonic Integrated Circuits (Wiley, New York, 1995).

${ }^{2}$ J. R. Baird, W. N. Carr, and B. S. Reed, Trans. AIME. 230, 286 (1964).

${ }^{3}$ P. M. Smowton and P. Blood, Appl. Phys. Lett. 70, 2365 (1997).

${ }^{4}$ A. Moritz, R. Wirth, C. Geng, F. Scholz, and A. Hangleiter, Appl. Phys. Lett. 68, 1217 (1996). 